\title{
Nanoconfinement and Mass Transport in Silica Mesopores: the Role of Charge at the Single Molecule and Single Pore Levels
}

\author{
Ruwandi Kumarasinghe, Takashi Ito* and Daniel A. Higgins* \\ Department of Chemistry, Kansas State University, 213 CBC Building, Manhattan, Kansas \\ 66506-0401, USA
}

Additional information on the precision of single molecule localization is given along with representative plots of single molecule $M S D$ values determined from representative trajectories for each of the four PDI dyes. The video data used in preparation of Figure 2 are also provided.

*Corresponding author emails: ito@ksu.edu and higgins@ksu.edu 


\section{Single Molecule Localization Precision}

The precision to which each fluorescent spot is calculated as follows: ${ }^{1}$

$$
\sigma^{2}=\frac{s^{2}+a^{2} / 12}{N}+\frac{8 \pi s^{4} b^{2}}{a^{2} N^{2}}
$$

In this equation, $s, a$, and $N$ represent the standard deviation of the point-spread function (i.e., the Gaussian width of each fluorescent spot), the pixel size, and the total photon counts recorded under the Gaussian spot, respectively. The $b^{2}$ term represents the background counts, which were taken as the variance of the measured counts in image regions free of fluorescent spots. Typical image parameters include $s=0.160 \mu \mathrm{m}, a=0.125 \mu \mathrm{m}, N=1000$ counts, and $b^{2}=138$ counts. These values yield an estimate of $\sim 13 \mathrm{~nm}$ for the localization precision, $\sigma$.

Single Molecule MSD Plots

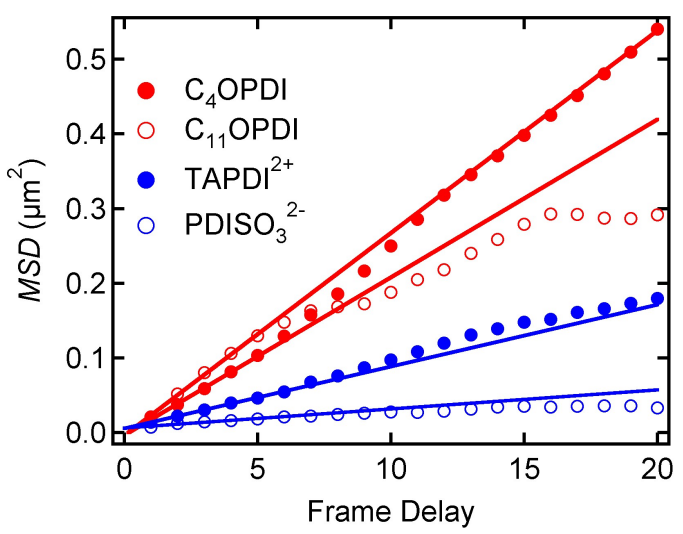

Figure S1. MSD plots (symbols) for the trajectories shown in Figure 3A,C,D,F. Each has been fit to eq 5 (solid lines) up to $n=5$. The results of the fits were used to determine $D_{M S D}$, giving values of $0.033 \mu \mathrm{m}^{2} / \mathrm{s}\left(\mathrm{C}_{4} \mathrm{OPDI}\right), 0.043 \mu \mathrm{m}^{2} / \mathrm{s}\left(\mathrm{C}_{11} \mathrm{OPDI}\right), 0.013 \mu \mathrm{m}^{2} / \mathrm{s}\left(\mathrm{TAPDI}^{2+}\right)$, and 0.004 $\mu \mathrm{m}^{2} / \mathrm{s}\left(\mathrm{PDISO}_{3}{ }^{2-}\right)$. 
Video S1. Fluorescence video (200 frames, $\sim 3$ frames/s) depicting $\mathrm{C}_{4}$ OPDI dye motion in a locally ordered region of a surfactant- and solvent-filled mesoporous silica film. The data shown in Figure 2a were derived from this video.

Video S2. Fluorescence video (200 frames, $\sim 3$ frames/s) depicting TAPDI ${ }^{2+}$ dye motion in a locally ordered region of a surfactant- and solvent-filled mesoporous silica film. The data shown in Figure $2 b$ were derived from this video.

Video S3. Fluorescence video (200 frames, $\sim 3$ frames/s) depicting $\mathrm{PDISO}_{3}{ }^{2-}$ dye motion in a locally ordered region of a surfactant- and solvent-filled mesoporous silica film. The data shown in Figure 2c were derived from this video.

\section{References}

(1) Thompson, R. E.; Larson, D. R.; Webb, W. W. Precise Nanometer Localization Analysis for Individual Fluorescent Probes. Biophys. J. 2002, 82, 2775-2783. 